\title{
Relating different cycle spaces of the same infinite graph
}

\author{
R. Bruce Richter and Brendan Rooney \\ Submitted: Jul 5, 2009; Accepted: Jun 11, 2001; Published: Jun 21, 2011 \\ Mathematics Subject Classificiation: 05C10
}

\begin{abstract}
Casteels and Richter have shown that if $X$ and $Y$ are distinct compactifications of a locally finite graph $G$ and $f: X \rightarrow Y$ is a continuous surjection such that $f$ restricts to a homeomorphism on $G$, then the cycle space $Z_{X}$ of $X$ is contained in the cycle space $Z_{Y}$ of $Y$. In this work, we show how to extend a basis for $Z_{X}$ to a basis of $Z_{Y}$.
\end{abstract}

\section{Introduction}

Bonnington and Richter [1] introduced the cycle space of a locally finite graph as the edge sets of subgraphs in which every vertex has even degree. Diestel and Kühn [4] introduced a different cycle space for a locally finite graph $G$ as the space "generated" by embeddings of circles into the Freudenthal compactification $\mathbb{F}(G)$ of $G$. (The space $\mathbb{F}(G)$ is obtained from $G$ by adding one "point at infinity" for each "end" of $G$.)

Vella and Richter [7] unified these notions by introducing edge spaces and showing that a nice cycle space theory holds for compact, weakly Hausdorff edge spaces (for definitions see Section 2). In particular, the cycle space of Bonnington and Richter can be viewed as the Diestel-Kühn cycle space, but in Alexandroff's 1-point compactification $\mathbb{A}(G)$ of the locally finite graph $G$, rather than in $\mathbb{F}(G)$.

Our first principal result clarifies the relation between the cycle spaces of a given edge space, and its image under a continuous edge-preserving map. In particular, we show how to use spanning trees to extend a basis of the smaller space to a basis of the larger space. This recalls the fact that a basis of the cycle space of a given finite graph $G$ can be extended to a basis of the cycle space of any graph $G^{\prime}$ resulting from a sequence of vertex identifications of $G$.

In order to better comprehend the statement, loosely speaking an edge space $(X, E)$ consists of a topological space $X$ with a specified set $E$ of disjoint open arcs (each arc also an open set in $X), Z_{(X, E)}$ denotes the cycle space of $(X, E)$ (its elements are subsets of $E)$, and, relative to an analogue $T$ of a spanning tree for $(X, E)$, for each $e \in E \backslash T$, $C_{T}(e)$ is the fundamental cycle contained in $T \cup e$. 
Theorem 1.1 Let $(X, E)$ and $(Y, E)$ be compact, connected, weakly Hausdorff edge spaces and let $f: X \rightarrow Y$ be a continuous surjection so that, for each $e \in E, f(e)=e$ and $f(X \backslash E)=Y \backslash E$. Let $T_{Y}$ be the edge set of a spanning tree of $(Y, E)$. Let $T_{X}$ be the edge set of a spanning tree of $(X, E)$ for which $T_{Y} \subseteq T_{X}$ and let $z$ be in $Z_{(Y, E)}$. Then there exist unique $z^{\prime} \in Z_{(X, E)}$ and, for each $e \in T_{X} \backslash T_{Y}, \alpha_{e} \in\{0,1\}$ so that $z=z^{\prime}+\sum_{e \in T_{X} \backslash T_{Y}} \alpha_{e} C_{T_{Y}}(e)$.

A main result in [1] considers a locally finite graph $G$ embedded in the sphere with a finite number of accumulation points. If there are $k+1$ accumulation points, then the face boundaries generate a subspace of the cycle space of $\mathbb{A}(G)$ having index $k$. Our other principal result generalizes this fact. If the graph $G$ can be embedded in the sphere with $k+1$ accumulation points, performing $k$ identifications converts the closure of the graph into $\mathbb{A}(G)$. The theorem below treats the abstract case of a single identification, while the corollary, proved by a trivial induction from the theorem, provides the case of finitely many identifications.

Theorem 1.2 Let $(X, E)$ be a compact, connected, weakly Hausdorff edge space such that $X \backslash E$ is totally disconnected. Let $x, y \in X \backslash E$ be distinct and let $Y$ be the quotient space obtained from $X$ by identifying $x$ and $y$. Let $P$ be the edge set of an xy-path in $X$. Then $P \in Z_{(Y, E)} \backslash Z_{(X, E)}$ and, for each $z \in Z_{(Y, E)} \backslash Z_{(X, E)}$, there is a unique element $z^{\prime} \in Z_{(X, E)}$ so that $z=z^{\prime}+P$.

Corollary 1.3 Let $(X, E)$ be a compact, connected, weakly Hausdorff edge space such that $X \backslash E$ is totally disconnected. For $i=1,2, \ldots, k$, let $\left\{x_{i}, x_{i}^{\prime}\right\}$ be pairs of elements of $X \backslash E$ so that, for any nonempty subset $I$ of $\{1,2, \ldots, k\},\left|\cup_{i \in I}\left\{x_{i}, x_{i}^{\prime}\right\}\right|>|I|$. Let $Y$ be the quotient space obtained from $X$ by identifying $x_{i}$ with $x_{i}^{\prime}, i=1,2, \ldots, k$. For each $i=1,2, \ldots, k$, let $P_{i}$ be an $x_{i} x_{i}^{\prime}$-path in $X$. If $z \in Z_{(Y, E)}$, then there exist unique $z^{\prime} \in Z_{(X, E)}$ and, for $i=1,2, \ldots, k, \alpha_{i} \in\{0,1\}$ so that $z=z^{\prime}+\sum_{i=1}^{k} \alpha_{i} P_{i}$.

We remark that Theorem 1.2 and Corollary 1.3 apply in particular to the case of distinct compactifications of a graph $G$; we are also motivated by other spaces. This point is discussed in Section 3. All of the relevant definitions and preliminary results can be found in Section 2. Section 4 has the proof of Theorem 1.1, while Theorem 1.2 is

proved in Section 6. Section 5 is devoted to a deeper understanding of cycles that is used in Section 6.

\section{Preliminaries/Definitions}

In this section, we provide the background material to place our work in context. We believe there is merit to treating our infinite spaces more combinatorially than would be usual in topology. Thus, we prefer edges to be open singletons, rather than real intervals. In many places in this work, the vertex set will consist of closed singletons making a totally disconnected subspace - this setting provides a very natural, simultaneous generalization 
of finite and infinite graphs, as well as the Freudenthal compactification (defined below) of a locally finite graph.

An edge space is an ordered pair $(X, E)$ consisting of a topological space $X$ and a subset $E$ of $X$, called the edges, so that each $e \in E$ is an open singleton whose boundary has at most two elements. We refer to the points in the boundary of $e$ as the nodes of $e$ and, if $v$ is a node of $e$, then we say that $v$ and $e$ are incident. The motivation for edge spaces is to generalize a natural topology of a graph. Let $G$ be a graph with vertex set $V$ and edge set $E$. In the classical topology [7], the basic open sets are: for each $e \in E$, $\{e\}$; and, for each $v \in V, v$ plus all its incident edges. With this topology, topological and graph-theoretic connection coincide. Among other things, we will be interested in compactifications of $G$, which, for infinite graphs, means adding some additional points and open sets corresponding to the "ends" of $G$.

An end of a graph $G$ consists of an equivalence class of rays: two rays $R$ and $S$ are equivalent if, for every finite set $W$ of vertices, $R$ and $S$ have their tails in the same component of $G-W$. A basic neighbourhood of the end $\omega$ is the component $K$ of $G-W$ containing the tails of the rays in $\omega$, plus all edges between $K$ and $W$ - but not their incident vertices from $W$ - and all the other ends whose rays' tails lie in $K$. The Freudenthal compactification $\mathbb{F}(G)$ of $G$ is obtained by adding a new point for each end, together with the basic neighbourhoods for each end. If $G$ is locally finite, then $\mathbb{F}(G)$ is compact. (More generally, if the deletion of any finite set of vertices leaves only finitely many components, then $\mathbb{F}(G)$ is compact.) A compactification of $G$ is any space obtained by partitioning the end set of $G$ into closed sets, and identifying the points in $\mathbb{F}(G)$ corresponding to each part.

Since most topology texts generally assume all singletons are closed for their more advanced theorems, at this stage it is necessary to develop the topological results more suited to our context. This leads us to the following natural extension of the Hausdorff property. A space $X$ is weakly Hausdorff if for all $x, y \in X$ there exist open sets $U_{x}$ and $U_{y}$ so that: $x \in U_{x} ; y \in U_{y}$; and $\left|U_{x} \cap U_{y}\right|$ is finite. In the classical topology on a graph, the only pairs that have no disjoint neighbourhoods are adjacent vertices, and a vertex with an incident edge.

A cycle in an edge space $(X, E)$ is a connected subspace $Y$ of $X$ so that: $Y \cap E$ is not empty; for every $e \in Y \cap E, Y \backslash\{e\}$ is connected; and, for any distinct $e, f \in Y \cap E$, $Y \backslash\{e, f\}$ is not connected. This definition should be compared with the "embedding of a circle" definition of Diestel and Kühn. In Section 5, we clarify the relation between these two definitions.

The cycle space $Z_{(X, E)}$ of $(X, E)$ is the smallest subset of the power set $2^{E}$ of $E$ that contains all the (edge-sets of) cycles and is closed under thin sums: a set $\mathcal{C} \subseteq 2^{E}$ is thin if each element of $E$ occurs in only finitely many elements of $\mathcal{C}$ and the "sum" is the set of elements of $E$ that are in an odd number of elements of $\mathcal{C}$.

We assume the reader is familiar with the following results from [7].

Lemma 2.1 Let $(X, E)$ be a compact, weakly Hausdorff edge space.

1. There is a minimal connected subset $T$ of $X$ containing $X \backslash E$. 
2. Each edge e in $E \backslash T$ is in a unique cycle, with edge set $C_{T}(e)$, contained in ( $E \cap$ $T) \cup\{e\}$.

3. The set $\mathcal{C}=\left\{C_{T}(e) \mid e \in E \backslash T\right\}$ is thin and, for every element $z$ of $Z_{(X, E)}$, $z=\sum_{e \in z \backslash T} C_{T}(e)$.

The minimal connected set $T$ from Lemma 2.1 (1) is defined to be a spanning tree of $(X, E)$ (note that if $X \backslash E$ is not totally disconnected, then $T$ may not resemble a tree). The elements $C_{T}(e)$ of $\mathcal{C}$ in (3) are defined to be the fundamental cycles (with respect to $T)$. We shall also make use of the following results from Casteels and Richter [2].

Lemma 2.2 Let $(X, E)$ and $(Y, F)$ be compact, weakly Hausdorff edge spaces and let $f$ : $X \rightarrow Y$ be a continuous surjection so that $f: E \rightarrow F$ is a bijection and $f(X \backslash E)=Y \backslash F$. Then:

1. for any spanning tree $T_{Y}$ of $(Y, F)$, there is a spanning tree $T_{X}$ of $(X, E)$ containing $f^{-1}\left(T_{Y}\right) ;$ and

2. identifying each $e \in E$ with $f(e), Z_{(X, E)} \subseteq Z_{(Y, F)}$.

Our first result, Theorem 1.1, further clarifies the relationship $Z_{(X, E)} \subseteq Z_{(Y, F)}$ of Lemma 2.2 by showing (perhaps as expected) that the basis $\left\{C_{T_{X}}(e): e \in E \backslash T_{X}\right\}$ of $Z_{(X, E)}$ can be extended to a basis of $Z_{(Y, F)}$ by adding precisely the fundamental cycles $C_{T_{Y}}(f(e))$, for $e \in E \cap T_{X} \backslash f^{-1}\left(T_{Y}\right)$.

\section{$3 \quad$ Examples}

In this section, we show that the hypotheses of Theorem 1.2 and Corollary 1.3 hold in cases of interest.

As a simple example, consider the 2 -way infinite ladder $L$ (see Figure 1). Let $a$ be the end of $L$ to the left and $b$ the end to the right. Then $\mathbb{F}(L)$ is $L$ plus these two additional points. Let $L_{a=b}$ denote the space obtained from $\mathbb{F}(L)$ by identifying $a$ and $b$ (this is the 1-point compactification $\mathbb{A}(L)$ of $L$ ). Let $v$ and $w$ be any two vertices of $L$. We can set $L_{a=v}$ to be the space obtained by identifying $a$ with $v, L_{b=w}$ the space obtained from $\mathbb{F}(L)$ by identifying $b$ with $w$, and $L_{a=v, b=w}$ the space obtained from $L_{a=v}$ by identifying $b$ with $w$. We emphasize that the compact spaces $L_{a=v}$ and $L_{b=w}$ are not compactifications of $L$. However, they are compact spaces containing (although not homeomorphically) the original graph and illustrate the notion of vertex identification. They are "graph-like continuua," defined below.

All these spaces have cycle spaces, which we naturally denote by $Z_{\mathbb{F}}, Z_{a=b}, Z_{a=v}, Z_{b=w}$, and $Z_{a=v, b=w}$. All of these contain $Z_{\mathbb{F}}$, but, for example, none of $Z_{a=b}, Z_{a=v}$, and $Z_{b=w}$ is contained in another of these three, while the last two of these three are both contained in $Z_{a=v, b=w}$. All the containments mentioned have index 1 except $Z_{\mathbb{F}}$ has index 2 in $Z_{a=v, b=w}$. 


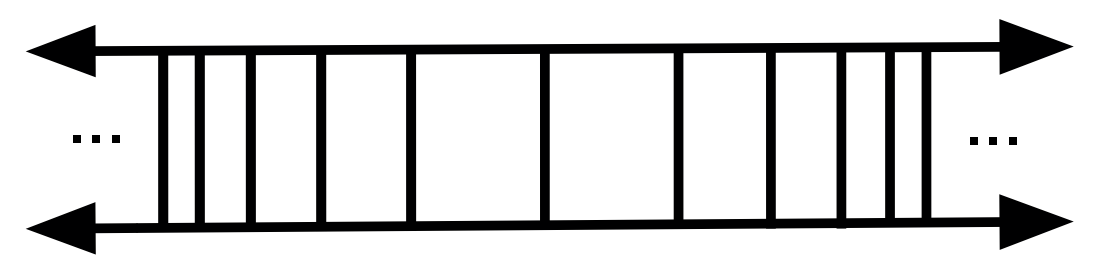

Figure 1: 2-way infinite ladder

In all cases, when we delete the set of edges from the superspace of $L$, the result is a Hausdorff subspace. This recalls the fact that if $f: X \rightarrow Y$ is a continuous surjection from a compact space $X$ to a Hausdorff space $Y$, then $f$ is a quotient map. In our context, $Y$ is not Hausdorff. Here is a small generalization of the standard fact which is more appropriate for our context. This is intended as a comment and will not be used in this work.

Lemma 3.1 Let $(X, E)$ be a compact edge space and let $(Y, F)$ be a weakly Hausdorff edge space such that $Y \backslash F$ is Hausdorff. Let $f: X \rightarrow Y$ be a continuous function for which: $f: E \rightarrow F$ is a bijection; for $e \in E, \operatorname{Cl}(f(e))=f(\operatorname{Cl}(e))$; and $f(X \backslash E)=Y \backslash F$. Then $f$ is a quotient map.

We remark that if $(Y, F)$ is weakly Hausdorff, then $Y \backslash F$ is also weakly Hausdorff. A weakly Hausdorff space $X$ is Hausdorff if and only if $X$ is $T_{1}$, which in turn is equivalent to: every point in $X$ is closed. Thus, the assumption in Lemma 3.1 that $Y \backslash F$ is Hausdorff can be "weakened" to the assumption that each point in $Y \backslash F$ is closed. It would be interesting to know if there is a generalization of Lemma 3.1 that does not require the assumption that $Y \backslash F$ is Hausdorff. The omitted proof of Lemma 3.1 is not difficult.

Another example of spaces to which Theorem 1.2 and Corollary 1.3 apply is the class of graph-like spaces introduced by Thomassen and Vella [6]. We consider a minor variation on these that are of specific interest to us. A graph-like continuum is an edge space $(X, E)$ for which $X$ is compact and connected, $E$ is countable, and $X \backslash E$ is totally disconnected and Hausdorff. These spaces include compactifications of locally finite graphs and the space $L_{a=v}$ described above. Thomassen and Vella [6] show that these spaces satisfy a form of Menger's Theorem. They have also been shown to satisfy a version of MacLane's Theorem by Christian, Richter and Rooney [3]. As such, they provide a natural class of spaces that generalize finite graphs.

We conclude with two simple examples. For the first example, let $X_{1}$ be the space consisting of continuumly many points with the discrete topology. Let $X$ be the space obtained from $X_{1}$ by adding two new points $x$ and $y$, each joined by an edge to each point of $X_{1}$. Let $Y$ be the space consisting of the real line $\mathbb{R}$, plus one new point $\alpha$, joined by parallel edges $e_{1, r}, e_{2, r}$ to each point $r$ in $\mathbb{R}$. If $b: X_{1} \rightarrow \mathbb{R}$ is any bijection, then $b$ is 
continuous, as is the function $f: X \rightarrow Y$ defined by

$$
f(t)= \begin{cases}b(t) & t \in X_{1} \\ \alpha & t=x, y \\ e_{1, r} & t \text { is an edge incident with } x \text { and } x^{\prime}, \text { and } b\left(x^{\prime}\right)=r \\ e_{2, r} & t \text { is an edge incident with } y \text { and } x^{\prime}, \text { and } b\left(x^{\prime}\right)=r .\end{cases}
$$

The map $f$ is clearly not a quotient map and $X$ is not compact. The cycle space of $X$ consists of all sets $z$ of edges such that, for each pair of edges $e, e^{\prime}$ incident with a common vertex of $X_{1}$, either both or neither of $e$ and $e^{\prime}$ are in $z$. On the other hand, the cycle space of $Y$ consists of all subsets of the set of edges. (For example, if, for each non-negative integer $i, e_{1, i}$ is incident with $i \in \mathbb{R}$, then $e_{1,0}=\sum_{i \geq 0}\left\{e_{1, i}, e_{1, i+1}\right\}$ and $\left\{e_{1, i}, e_{1, i+1}\right\}$ is the edge-set of a cycle in $Y$.) Thus $Z_{(X, E)}$ has infinite index in $Z_{(Y, E)}$.

Our other example is a graph $G$ with three vertices $u, v$ and $w$ so that the pairs of vertices $\{u, v\}$ and $\{v, w\}$ are each joined by a countable infinity of parallel edges. We use the classical topology, so that $G$ is compact. In this case, if $e_{0}$ is an edge of $G$, then, for any infinite sequence $\left\{e_{n}\right\}_{n \geq 0}$ of distinct edges, $\left\{e_{0}\right\}$ is the sum of the circuits $\left\{e_{i}, e_{i+1}\right\}$, $i=0,1,2, \ldots\}$; thus $Z_{G}$ consists of all subsets of edges. If we identify $u$ with $w$ to get $(Y, E)$, then $Z_{(Y, E)}$ is still all subsets of $E$. Thus some condition, such as weakly Hausdorff, is required in Theorem 1.2 in order to grow the cycle space after the identification.

\section{Proof of Theorem 1.1}

In this section we prove Theorem 1.1. To this end, let $z \in Z_{(Y, E)}$, let $T_{Y}$ be the edge set of a spanning tree of $(Y, E)$ and let $T_{X}$ be the edge set of a spanning tree of $(X, E)$ so that $T_{Y} \subseteq T_{X}$.

The fundamental cycles $C_{T_{Y}}(e)$, with respect to $T_{Y}$, generate the cycle space $Z_{(Y, E)}$. Thus, for $z \in Z_{(Y, E)}$, we have $z=\sum_{e \in z \backslash T_{Y}} C_{T_{Y}}(e)$. We would like to express this in terms of the fundamental cycles of $T_{X}$. Consider $e \in E \backslash T_{Y}$. Then either $e \in T_{X} \backslash T_{Y}$ or $e \in E \backslash T_{X}$. We partition $E \backslash T_{X}$ into $E_{d}$ and $E_{s}: E_{d}$ consists of those $e \in E \backslash T_{X}$ having its nodes in distinct components of $T_{Y}$, while $E_{s}$ consists of those $e \in E \backslash T_{X}$ having both nodes in the same component of $T_{Y}$.

We claim that, for $e \in E_{s}, C_{T_{X}}(e)=C_{T_{Y}}(e)$. To see this, note that $C_{T_{X}}(e) \in Z_{(X, E)}$, so that Lemma 2.2 implies $C_{T_{X}}(e) \in Z_{(Y, E)}$. Therefore, Lemma 2.1 implies $C_{T_{X}}(e)=$ $\sum_{e^{\prime} \in C_{T_{X}}(e) \backslash T_{Y}} C_{T_{Y}}\left(e^{\prime}\right)$. But, for example by Lemma 10 in [7], $C_{T_{X}}(e) \subseteq T_{Y} \cup\{e\}$, so $C_{T_{X}}(e) \subseteq T_{Y} \cup\{e\}$, and, therefore, $C_{T_{X}}(e) \backslash T_{Y}$ contains only $\{e\}$. Thus, $C_{T_{X}}(e)=C_{T_{Y}}(e)$, as required.

For $e \in E_{d}$, we have that $C_{T_{X}}(e)$ contains edges in $T_{X} \backslash T_{Y}$, so that $C_{T_{X}}(e)=$ $\sum_{e^{\prime} \in C_{T_{X}}(e) \backslash T_{Y}} C_{T_{Y}}\left(e^{\prime}\right)$, and the sum has more than one summand. So we have

$$
z=\sum_{e \in z \backslash T_{Y}} C_{T_{Y}}(e)=\sum_{e \in z \cap E_{s}} C_{T_{X}}(e)+\sum_{e \in z \cap E_{d}} C_{T_{Y}}(e)+\sum_{e \in z \cap\left(T_{X} \backslash T_{Y}\right)} C_{T_{Y}}(e) .
$$

We analyze $\sum_{e \in z \cap E_{d}} C_{T_{Y}}(e)$. 
We saw above that, for $e \in E_{d}$,

$$
C_{T_{X}}(e)=\sum_{e^{\prime} \in C_{T_{X}}(e) \backslash T_{Y}} C_{T_{Y}}\left(e^{\prime}\right)=C_{T_{Y}}(e)+\sum_{e^{\prime} \in T_{X} \cap C_{T_{X}}(e) \backslash T_{Y}} C_{T_{Y}}\left(e^{\prime}\right) .
$$

Rearranging,

$$
C_{T_{Y}}(e)=C_{T_{X}}(e)+\sum_{e^{\prime} \in T_{X} \cap C_{T_{X}}(e) \backslash T_{Y}} C_{T_{Y}}\left(e^{\prime}\right) .
$$

Summing over $e \in z \cap E_{d}$ yields

$$
\sum_{e \in z \cap E_{d}} C_{T_{Y}}(e)=\sum_{e \in z \cap E_{d}} C_{T_{X}}(e)+\sum_{e \in z \cap E_{d}}\left(\sum_{e^{\prime} \in T_{X} \cap C_{T_{X}}(e) \backslash T_{Y}} C_{T_{Y}}\left(e^{\prime}\right)\right) .
$$

We claim that $\sum_{e \in z \cap E_{d}}\left(\sum_{e^{\prime} \in T_{X} \cap C_{T_{X}}(e) \backslash T_{Y}} C_{T_{Y}}\left(e^{\prime}\right)\right)$ is defined. To see this, note that Lemma 2.1 implies $\left\{C_{T_{X}}(e) \mid e \in E \backslash T_{X}\right\}$ is thin, so each $e^{\prime}$ occurs in only finitely many of the $T_{X} \cap C_{T_{X}}(e)$, for $e \in z \cap E_{d}$. Thus, each $C_{T_{Y}}\left(e^{\prime}\right)$ occurs only finitely often and, since $\left\{C_{T_{Y}}\left(e^{\prime}\right) \mid e^{\prime} \in E \backslash T_{Y}\right\}$ is thin, every edge occurs in only finitely many terms in

$$
\sum_{e \in z \cap E_{d}}\left(\sum_{e^{\prime} \in T_{X} \cap C_{T_{X}}(e) \backslash T_{Y}} C_{T_{Y}}\left(e^{\prime}\right)\right)
$$

It follows that

$$
\begin{aligned}
z= & \sum_{e \in z \cap E_{s}} C_{T_{X}}(e)+\sum_{e \in z \cap T_{X} \backslash T_{Y}} C_{T_{Y}}(e)+\sum_{e \in z \cap E_{d}} C_{T_{Y}}(e) \\
= & \sum_{e \in z \cap E_{s}} C_{T_{X}}(e)+\sum_{e \in z \cap T_{X} \backslash T_{Y}} C_{T_{Y}}(e)+\sum_{e \in z \cap E_{d}} C_{T_{X}}(e) \\
& +\sum_{e \in z \cap E_{d}}\left(\sum_{e^{\prime} \in T_{X} \cap C_{T_{X}}(e) \backslash T_{Y}} C_{T_{Y}}\left(e^{\prime}\right)\right) .
\end{aligned}
$$

We comment that all four sums in the last equation are over thin families, so the entire expression is over a thin family. We note that the first and third of these sums yield elements of $Z_{(X, E)}$, while the other two sums are linear combinations of the cycles $C_{T_{Y}}(e)$, for $e \in T_{X} \backslash T_{Y}$, so this last sum for $z$ can be rewritten as

$$
z=z^{\prime}+\sum_{e \in T_{X} \backslash T_{Y}} \alpha_{e} C_{T_{Y}}(e)
$$

with $z^{\prime} \in Z_{(X, E)}$ as claimed.

To prove uniqueness, suppose $z^{\prime \prime} \in Z(X, E)$ and $\alpha_{e}^{\prime} \in\{0,1\}$ are such that we also have

$$
z=z^{\prime \prime}+\sum_{e \in T_{X} \backslash T_{Y}} \alpha_{e}^{\prime} C_{T_{Y}}(e) .
$$


Then

$$
z^{\prime}+z^{\prime \prime}=\sum_{e \in T_{X} \backslash T_{Y}}\left(\alpha_{e}+\alpha_{e}^{\prime}\right) C_{T_{Y}}(e) .
$$

The left-hand side of this equation is an element of $Z(X, E)$, while the right-hand side is a subset of $T_{X}$. The only element of $Z(X, E)$ contained in a spanning tree of $X$ is the empty set, from which we conclude that $z^{\prime}=z^{\prime \prime}$. Since each element of $Z(Y, E)$ is uniquely expressible as a sum of fundamental cycles, this further implies that, for $e \in T_{X} \backslash T_{Y}$, $\alpha_{e}=\alpha_{e}^{\prime}$.

\section{Cycles}

Before we can prove Theorem 1.2, we need to prove some general facts about cycles. The main point of this section is to prove that, in the special case of a compact, weakly Hausdorff edge space $(X, E)$ with $X \backslash E$ totally disconnected (which holds for the Freudenthal compactification of a connected, locally finite graph, or for Diestel and Kühn's identification space $\tilde{G}$ - the identifications are required to make $\mathbb{F}(G) \backslash E$ totally disconnected - in the case $G$ is 2-connected and no two vertices are joined by infinitely many edgedisjoint paths [5]), the edge-set of a cycle determines the cycle and, furthermore, no cycle is contained in another cycle. These issues have been considered in various guises, such as the discussion in [5, p. 838] or [7, Thm. 35].

In spaces that have intervals for edges (i.e. for the spaces considered in $[1,4,5,6]$ ) some of this material follows more naturally. Given an edge space $(X, E)$ having $X \backslash E$ totally disconnected, the most natural topological space arising from $X$ in which every edge is an interval is that space $X^{\prime}$ consisting of the points of $X \backslash E$ and an open interval $\gamma_{e}$ for each $e \in E$. Each open set $U$ in $X$ yields the open set $(U \backslash E) \cup\left\{\gamma_{e} \mid e \in U \cap E\right\}$ in $X^{\prime}$. These open sets, together with the open subintervals of the $\gamma_{e}$ make the basic open sets for $X^{\prime}$.

If, in addition, $(X, E)$ is a compact, weakly Hausdorff edge space, then $X^{\prime}$ is a compact, Hausdorff space. It is now not hard to show that if $C$ is a cycle in $(X, E)$ and $C \cap E$ is countable, then $(C \backslash E) \cup\left\{\gamma_{e} \mid e \in C \cap E\right\}$ is the homeomorphic image of a circle. The cyclic sequence of edges in $C$ tells us how to place the intervals $\gamma_{e}$ around the circle; the fact that $C$ is closed (Theorem 5.2) shows that the points of $C \backslash E$ go in the right places to make the map to the circle a homeomorphism. This generalizes [7, Thm. 35], without the assumption that $X$ is metric. In this context, the desired properties of cycles follow easily.

To see why this method does not work at our level of generality, consider the following example. Let $\alpha$ be the smallest uncountable ordinal. For each ordinal $\beta<\alpha$, we include the edge $e_{\beta}$ having nodes $\beta$ and $\beta+1$. We also include an edge $e_{\alpha}$ joining $\alpha$ and 0 . The basic open sets are: the singletons $e_{\beta}, \beta \leq \alpha$; for each non-limit ordinal $\beta,\left\{e_{\beta-1}, \beta, e_{\beta}\right\}$; and, for each limit ordinal $\beta \leq \alpha$ and each ordinal $\gamma<\beta,\{\delta \mid \gamma<\delta \leq \beta\} \cup\left\{e_{\delta} \mid \gamma \leq \delta \leq \beta\right\}$. The resulting edge space is compact, weakly Hausdorff and is a cycle having uncountably many edges. This cannot possibly correspond to the homeomorphic image of a circle. 
We begin with an easy result.

Lemma 5.1 Let $(X, E)$ be a compact edge space so that $X \backslash E$ is totally disconnected. Let $F \subseteq E$ and let $x \in X \backslash E$ be such that $x \notin \mathrm{Cl}(F)$. Then $x$ is a component of $(X \backslash E) \cup F$.

Proof. For each $y \in \mathrm{Cl}(F) \backslash F$, let $U_{y}$ and $W_{y}$ be open sets in $X$ so that $\left(U_{y} \backslash E, W_{y} \backslash E\right)$ is a separation of $X \backslash E$ with $x \in U_{y}$ and $y \in W_{y}$. The sets $W_{y}$ form an open cover of the compact set $\mathrm{Cl}(F)$ and, therefore, there is a finite subcover $W_{y_{1}}, W_{y_{2}}, \ldots, W_{y_{k}}$. Let $W=\bigcup_{i=1}^{k} W_{y_{i}}$ and let $Z=\bigcap_{i=1}^{k} U_{y_{i}}$.

We observe that $\mathrm{Cl}(F) \subseteq W$ and that $Z^{\prime}=Z \backslash \mathrm{Cl}(F)$ is open. Furthermore $x \in Z^{\prime}$ and $\left(W, Z^{\prime}\right)$ is a separation of $(X \backslash E) \cup F$. Therefore, the component of $(X \backslash E) \cup F$ containing $x$ is contained in $Z^{\prime} \backslash E$ and, therefore, is $x$.

Our next step is to show that cycles are closed. We wonder if there is a simpler proof of this fact. The reader might wish to compare this theorem with the example [7, p. 137] of a cycle, in a compact, weakly Hausdorff edge space, that is not closed and with the fact that the edges in a circle are required by Diestel and Kühn to be dense in the circle $[4$, p. $74(3)]$ and $[5$, p. $838(1)]$.

Lemma 5.2 Let $(X, E)$ be a compact, weakly Hausdorff edge space so that $X \backslash E$ is totally disconnected. If $C$ is a cycle in $(X, E)$, then $C=\operatorname{Cl}(C \cap E)$.

Proof. We prove $C$ is closed; the result then follows from Lemma 5.1. Fix $e^{*} \in E \cap C$ and let $Y=C \backslash e^{*}$. Clearly $\mathrm{Cl}(C)=\mathrm{Cl}(Y) \cup \mathrm{Cl}\left(e^{*}\right)$. Since, for each $e \in E \cap C, \mathrm{Cl}(e) \subseteq C$, it follows that $C$ is closed if and only if $Y$ is closed, so we prove the latter.

By way of contradiction, suppose there is an $x \in \operatorname{Cl}(Y) \backslash Y$. Let $a$ and $b$ be the nodes of $e^{*}$ and notice that, for each $e \in E \cap Y, a$ and $b$ are in distinct components $K_{a}^{e}$ and $K_{b}^{e}$, respectively, of $Y \backslash e$. Set $A=\left\{e \in E \cap Y \mid x \in \mathrm{Cl}\left(K_{a}^{e}\right)\right\}$ and let $B=(E \cap Y) \backslash A$. Evidently, if $e \in B$, then $x \in \mathrm{Cl}\left(K_{b}^{e}\right)$.

We claim that $A \neq \varnothing$ and $B \neq \varnothing$. The proofs are similar, so we prove only $B \neq \varnothing$. Otherwise, for every $e \in E \cap Y, x \in \mathrm{Cl}\left(K_{a}^{e}\right)$. Thus, $\bigcap_{e \in E \cap Y} \mathrm{Cl}\left(K_{a}^{e}\right)$ is closed, connected, contains $a$ and $x$, and contains no edge of $X$. This contradicts the assumptions that $X \backslash E$ is totally disconnected and $x \notin Y$.

Next, observe that, if $e \in A$ and $e^{\prime} \in B$, then $x$ is in $\operatorname{Cl}\left(K_{a}^{e}\right) \backslash \mathrm{Cl}\left(K_{a}^{e^{\prime}}\right)$, and so $e \in K_{b}^{e^{\prime}}$. We now set $J=\bigcap_{e \in A} \mathrm{Cl}\left(K_{a}^{e}\right)$. Note that $x \in J$ and the previous sentence implies $B=J \cap E$. From an earlier remark, $x \in \cap_{e \in B} \mathrm{Cl}\left(K_{b}^{e}\right)$. It is also straightforward to see that $A=E \cap \bigcap_{e \in B} \mathrm{Cl}\left(K_{b}^{e}\right)$.

For $e \in A$ and $e^{\prime} \in B$, let $K_{e, e^{\prime}}$ be the component of $Y \backslash\left\{e, e^{\prime}\right\}$ containing neither $a$ nor $b$. Set $L=\bigcap_{e, e^{\prime}} \mathrm{Cl}\left(K_{e, e^{\prime}}\right)$.

A main point of the proof is to show $L$ is just $x$. For $e \in A$ and $e^{\prime} \in B, x \notin \mathrm{Cl}\left(K_{a}^{e^{\prime}}\right)$, $x \in \mathrm{Cl}\left(K_{a}^{e}\right)$ and $K_{a}^{e}=K_{a}^{e^{\prime}} \cup\left\{e^{\prime}\right\} \cup K_{e, e^{\prime}}$. Since $x \notin \mathrm{Cl}\left(K_{a}^{e^{\prime}}\right) \cup \mathrm{Cl}\left(e^{\prime}\right)$, we deduce that $x \in \mathrm{Cl}\left(K_{e, e^{\prime}}\right)$. Therefore, $x \in L$.

It is easy to see that no edge of $X$ is in $L$. We claim $L$ is connected, from which it follows that $L$ is just a single point. Firstly, for $e \in A$, set $L_{e}=\bigcap_{e^{\prime} \in B} \mathrm{Cl}\left(K_{e, e^{\prime}}\right)$. 
This is the nested intersection of closed, connected sets all containing $x$ and so is closed, connected, and contains $x$. Likewise, $L=\bigcap_{e \in A} L_{e}$ is closed, connected, and contains $x$. That is, $L$ is just $x$.

We are now in a position to show that $Y$ is not connected, which is the final contradiction. The separation we are looking for is

$$
\left(\bigcup_{e \in A} K_{b}^{e}, \bigcup_{e \in B} K_{a}^{e}\right)
$$

so we must show its two sets are open and partition $Y$. The disjointness is trivial: for $e \in A$ and $e^{\prime} \in B, K_{b}^{e} \cap K_{a}^{e^{\prime}}=\varnothing$. Since $L$ is just $x$, it follows that $\bigcap_{e \in A, e^{\prime} \in B} K_{e, e^{\prime}}=\varnothing$, whence $Y=\left(\bigcup_{e \in A} K_{b}^{e}\right) \cup\left(\bigcup_{e \in B} K_{a}^{e}\right)$.

To see, for example, that $\bigcup_{e \in A} K_{b}^{e}$ is open in $Y$, note first that $K_{b}^{e} \cup e=Y \backslash K_{a}^{e}$, so $K_{b}^{e} \cup e$ is open in $Y$. Thus, it suffices to show that, for each $e \in A$, there is an $e^{\prime} \in A$ so that $K_{b}^{e} \cup e \subseteq K_{b}^{e^{\prime}}$. Suppose for the edge $e^{+} \in A$, there is no such $e^{\prime}$. Then the node of $e^{+}$in $K_{a}^{e^{+}}$is in $\bigcap_{e \in A, e^{\prime} \in B} K_{e, e^{\prime}}$, a contradiction.

Theorem 5.3 Let $(X, E)$ be a compact, weakly Hausdorff edge space with $X \backslash E$ totally disconnected and let $Y_{1}$ and $Y_{2}$ be distinct cycles in $(X, E)$. Then $E \cap Y_{1}$ is not a subset of $E \cap Y_{2}$.

We remark that the example [7, Fig. 2, p. 137] shows that we need the assumption that $X \backslash E$ is totally disconnected.

Proof. For $i=1,2$, Lemma 5.2 shows $Y_{i}=\operatorname{cl}\left(E \cap Y_{i}\right)$. If $E \cap Y_{1} \subseteq E \cap Y_{2}$, then there is an edge $e \in E \cap Y_{2} \backslash Y_{1}$ (as the $Y_{i}$ are distinct) and $Y_{1}=\mathrm{Cl}\left(E \cap Y_{1}\right) \subseteq \operatorname{Cl}\left(E \cap Y_{2}\right)=Y_{2}$. If $f$ is any edge of $Y_{1}$, with nodes $a$ and $b, a$ and $b$ are in distinct components of $Y_{2} \backslash\{e, f\}$. But then $a$ and $b$ are in distinct components of $Y_{1} \backslash f$, contradicting the assumption that $Y_{1} \backslash f$ is connected.

\section{Proof of Theorem 1.2}

In this section, we give a proof of Theorem 1.2. For two points $x, y$ of $X$, an $x y$-path is a minimal closed connected set containing $x$ and $y$.

Proof of Theorem 1.2. Let $(X, E)$ be a compact, weakly Hausdorff edge space with $X \backslash E$ totally disconnected and let $x$ and $y$ be distinct points of $X \backslash E$. Let $Y$ be the quotient space obtained from $X$ by identifying $x$ and $y$. That is, the function $f: X \rightarrow Y$ defined by $f(t)=t$ for $t \notin\{x, y\}$ and $f(x)=f(y)=\{x, y\}$ is a continuous surjection so that if $U \subseteq X$ is open in $X$ and either $U \cap\{x, y\}=\emptyset$ or $\{x, y\} \subseteq U$, then $f(U)$ is open in $Y$. 
Claim 1 Let $S$ be a subspace of $X$ with finitely many components so that $x, y \in S$ and some component of $S$ is disjoint from $\{x, y\}$. Then $f(S)$ is not connected.

Proof. The proof is standard. Let $C$ be a component of $S$ disjoint from $\{x, y\}$. Because there are only finitely many components, there are open sets $U_{1}, U_{2}$ in $X$ so that $U_{1} \cap S=C$ and $U_{2} \cap S=S \backslash C$. Since $x, y \notin U_{1}$ and $x, y \in U_{2}$, both $f\left(U_{1}\right)$ and $f\left(U_{2}\right)$ are open in $Y$ and $f(S) \cap f\left(U_{1}\right)$ and $f(S) \cap f\left(U_{2}\right)$ make a separation of $f(S)$.

Let $\widehat{P}$ be a minimal connected closed subspace of $X$ containing $x$ and $y$ such that $P=\widehat{P} \cap E$.

Claim $2 P$ is the edge set of a cycle in $(Y, E)$.

Proof. In $X$, the deletion of any edge $e$ of $P$ disconnects $\widehat{P}$, producing precisely two components (see [7, Lemma 8]), one containing $x$ and the other containing $y$. Thus, $f(\widehat{P} \backslash\{e\})$ is connected in $Y$. On the other hand, if $e$ and $e^{\prime}$ are distinct edges of $P$, then $\widehat{P} \backslash\left\{e, e^{\prime}\right\}$ has precisely three components. One of these is disjoint from $\{x, y\}$ and so Claim 1 shows $f\left(\widehat{P} \backslash\left\{e, e^{\prime}\right\}\right)$ is not connected. Therefore, $P$ is a cycle in $Y$, so $P \in Z_{(Y, E)}$.

Claim $3 P \notin Z_{(X, E)}$.

Proof. Suppose $P$ is in $Z_{(X, E)}$. Then $P$ is the edge-disjoint union of cycles $\left\{C_{\lambda}\right\}[7$, Thm. 14]. By the preceding claim, $P$ is a cycle in $(Y, E)$ and each of the cycles $C_{\lambda}$ is in $Z_{(Y, E)}$ and, therefore, is the edge disjoint union of cycles $\left\{C_{\lambda, \mu}\right\}$. But then each $C_{\lambda, \mu}$ is a cycle contained in $P$. By Theorem 5.3, there is only one $C_{\lambda, \mu}$ and, therefore, $P$ is a cycle in $(X, E)$. Lemma 5.2 implies $\widehat{P}=P \cap E$. But in the proof of the preceding claim we showed $\widehat{P} \backslash e$ is not connected, a contradiction.

Now let $T_{X}$ and $T_{Y}$ be edge sets of spanning trees $\widehat{T}_{X}$ and $\widehat{T}_{Y}$ of $X$ and $Y$, respectively, with $T_{Y} \subseteq T_{X}$. Because $P \in Z_{(Y, E)} \backslash Z_{(X, E)}$, Theorem 1.1 implies $T_{X}$ has at least one edge that is not in $T_{Y}$. We claim it has precisely one such edge.

If $T_{X}$ has edges $e, f$ not in $T_{Y}$, then $\widehat{T}_{X} \backslash\{e, f\}$ has precisely three components. By Claim $1, \widehat{T}_{Y} \backslash\{e, f\}$ is not connected in $Y$, a contradiction.

So let $e$ be the edge in $T_{X} \backslash T_{Y}$. By Theorem 1.1, there is a $z \in Z_{(X, E)}$ so that $P=$ $C_{T_{Y}}(e)+z$. Rearranging, $C_{T_{Y}}(e)=P+z$. Again by Theorem 1.1, if $z^{\prime \prime} \in Z_{(Y, E)} \backslash Z_{(X, E)}$, there is a unique $z^{\prime}$ so that $z^{\prime \prime}=C_{T_{Y}}(e)+z^{\prime}$, whence $z^{\prime \prime}=P+\left(z+z^{\prime}\right)$, completing the proof of Theorem 1.2.

The crucial point for the induction to prove Corollary 1.3 is that deleting $k$ edges in an $x y$-path produces a subset having exactly $k+1$ components. If $k \geq 1$, then $x$ and $y$ are in different components. 


\section{ACKNOWLEDGMENT}

We are grateful to a referee of an earlier version of this work for suggesting an alternative proof of Claim 3 in the proof of Theorem 1.2. Its incorporation led to Theorem 5.3. The referees also suggested several improvements to the presentation.

Both authors gratefully acknowledge the financial support of NSERC.

\section{References}

[1] C.P. Bonnington and R.B. Richter, Graphs embedded in the plane with a bounded number of accumulation points, J. Graph Theory 44 (2003), no. 2, 132-147.

[2] K. Casteels and R.B. Richter, The cycle and bond spaces of an infinite graph, J. Graph Theory 59 (2008), no. 2, 225-239.

[3] R. Christian, R.B. Richter and B. Rooney, The planarity theorems of MacLane and Whitney for graph-like continua, Electron. J. Combin. 17 (2010), no. 1, Research Paper 12 .

[4] R. Diestel and D. Kühn, On infinite cycles. I, Combinatorica 24 (2004), no. 1, 69-89.

[5] R. Diestel and D. Kühn, Topological paths, cycles and spanning trees in infinite graphs, European J. Combin. 25 (2004), no. 6, 835-862.

[6] C. Thomassen and A. Vella, Graph-like continua, augmenting arcs, and Menger's Theorem, Combinatorica 28 (2008), no. 5, 595-623.

[7] A. Vella and R.B. Richter, The cycle space of a topological space, J. Graph Theory 59 (2008), no. 2, 114-144. 